\title{
Typical 3-D localization of tumor remnants of WHO grade II hemispheric gliomas-lessons learned from the use of intraoperative high-field MRI control
}

\author{
Venelin M. Gerganov • Amir Samii • Lennart Stieglitz • \\ Mario Giordano • Wolf O. Luedemann • Madjid Samii • \\ Rudolf Fahbusch
}

Received: 5 July 2010 /Accepted: 1 December 2010 /Published online: 14 January 2011

(C) Springer-Verlag 2011

\begin{abstract}
Background Complete resection of grade II gliomas might prolong survival but is not always possible. The goal of the study was to evaluate the location of unexpected grade II gliomas remnants after assumed complete removal with intraoperative (iop) MRI and to assess the reason for their non-detection.

Methods Intraoperative MR images of 35 patients with hemispheric grade II gliomas, acquired after assumed complete removal of preoperatively segmented tumor/ tumor part, were studied for existence of unexpected tumor
\end{abstract}

\author{
V. M. Gerganov $\cdot$ A. Samii $\cdot$ L. Stieglitz $\cdot$ M. Giordano $\cdot$ \\ W. O. Luedemann $\cdot$ M. Samii $\cdot$ R. Fahbusch \\ International Neuroscience Institute, \\ Rudolf Pichlmayr Str. 4, \\ Hannover 30625, Germany \\ A. Samii \\ e-mail: amir@samii.org \\ L. Stieglitz \\ e-mail: stieglitz@ini-hannover.de \\ M. Giordano \\ e-mail: giordano@ini-hannover.de \\ W. O. Luedemann \\ e-mail: luedemann@ini-hannover.de \\ M. Samii \\ e-mail: samii@ini-hannover.de \\ R. Fahbusch \\ e-mail: fahlbusch@ini-hannover.de \\ V. M. Gerganov $(\triangle)$ \\ Department of Neurosurgery, \\ International Neuroscience Institute-Hannover, \\ Rudolf Pichlmayr Str. 4, \\ Hannover 30625, Germany \\ e-mail: gerganov@ini-hannover.de
}

remnants. Remnants location was classified in relation to tumor cavity in axial and vertical planes. The relation of remnants to retractor position and to surgeons' visual axis, and the role of neuronavigational accuracy and brain shift, was assessed.

Results Unexpected remnants were found in 16 patients (46\%). In $29.2 \%$, the reason was loss of neuronavigational accuracy. In $21 \%$, remnants were in that part of the resection cavity, where the retractor had been placed initially. In $17 \%$, they were deeply located and hidden by the retractor. In 13\%, remnants were hidden by the overlapping brain; and in $21 \%$, the reason was not obvious. In $75 \%$ of all temporomesial tumors, remnants were posterolateral to the resection cavity. Remnants detection with iopMRI and update of neuronavigational data allowed further removal in 14 of 16 cases. In two cases, remnant location precluded their removal.

Conclusions Distribution of tumor remnants of grade II gliomas tends to follow some patterns. Targeted attention to the areas of possible remnants could increase the radicality of surgery, even if intraoperative imaging is not performed.

Keywords Tumor remnant · Grade II glioma . Intraoperative MRI · Neuronavigation

\section{Introduction}

Growing evidence demonstrates that complete resection of grade II gliomas might be associated with prolonged survival $[16,19,26]$. Further, it may lead to alleviation of symptoms, better control of tumor-related epilepsy, and may postpone malignant transformation, with the resultant limitation of patient survival [7]. The extent of resection, 
assessed with volumetric analysis, was found to be inversely correlated with the likelihood of tumor recurrence, time to recurrence, and tumor dignity at the time of recurrence [3, 28]. Shaw et al. [27] found that in case the residual tumor is $<1 \mathrm{~cm}$, the 5 -year recurrence rate was $26 \%$; in case the residual was $1-2 \mathrm{~cm}$, the recurrence rate was $68 \%$; and in those with $>2-\mathrm{cm}$ residual, the rate was $89 \%$.

The complete resection, however, is not possible in all grade II gliomas due to the infiltration of highly eloquent cortical and subcortical areas or blood vessels, or tumor location in risky areas, such as the anterior perforated substance [10]. In case of tumors that are considered completely removable before surgery based on preoperative functional imaging, intraoperatively it may be difficult to discern the pathological from normal brain tissue [1]. Even an extensive resection, including the surrounding normal brain tissue, cannot always guarantee that the whole grade II gliomas will be removed.

Various tools for intraoperative control, such as anatomical and functional neuronavigation, ultrasound, intraoperative CT or MRI, allow for improved visualization of the tumor and its possible remnants. The most reliable one is the intraoperative high-field MRI (iopMRI), which allows direct visualization of tumor remnants and an update of neuronavigational dataset. Thus, it compensates for the brain shift and makes additional and safe tumor resection possible [14]. In a recently published study, Nimsky et al. [22] showed that iopMRI revealed complete tumor removal initially in just $20 \%$ of the cases. In $41 \%$ of those cases, in which complete removal was ultimately achieved, additional resections after the imaging control have been performed. This technique, however, is very expensive and logistically demanding, which limits its wider application.

Some authors evaluated the preoperatively available factors that might lead or predict an incomplete removal $[17,31]$ but a systematic analysis of the intraoperative reasons for such outcome has not been performed. In the current study, we evaluated the three-dimensional location of remnants of grade II gliomas after assumed complete removal by the surgeon with a high-field strength iopMRI. Our goal was to demonstrate the typical sites of remnants. In case their distribution follows some patterns, this knowledge could be of help to avoid incomplete resections even when operating without iopMRI control.

\section{Patients and methods}

Patient population

During a 2-year period, from February 2007 to February 2009, 200 patients have been operated at the INI-Brain Suite; 35 of them had hemispheric grade II gliomas. Twenty four were males, nine female; the age ranged from 13 to 49 years and the mean age was 34.9 years. In all cases, the goal of surgery was to achieve a targeted removal of the tumor and preserve the surrounding normal brain tissue as much as possible. Complete removal was defined as removal of the whole tumor at the level of MRI discrimination. In 18 cases (51\%), complete removal was considered possible before the surgery. In 17 cases (49\%), the tumor could be removed completely; in 11 without and in six with additional resections after intraoperative imaging control. Thirty-two of the patients $(91 \%)$ did not have any additional neurological deficit; two $(6 \%)$ had a temporary deficit that recovered over the hospital stay, and only one patient $(3 \%)$ had a permanent hemiparesis.

\section{Study design}

The data of the patients with hemispheric grade II gliomas, including clinical history and presenting symptoms, tumor characteristics, patient positioning, anesthesia, intraoperative image acquisition and findings, as well as the procedure-related morbidity and outcome, have been collected prospectively in a standardized database. Before each surgery, the surgeon estimated the tumor as completely or incompletely resectable, based on the available structural and functional imaging data. Manual segmentation of the tumor outline was performed by using the navigation software. During tumor removal, navigational control was used to guide tumor resection. The extent of resection was evaluated with navigation when the planned amount of tumor removal was achieved and documented with screenshots. The images, acquired after achieving the goals of surgery, have been studied by three neurosurgeons for existence of tumor remnants - an area around the resection cavity, visible on iopMRI but not expected by the surgeon. Only those findings have been included that have been proven histologically as tumor. In case more than one remnant was found in a patient, they were analyzed separately. These data were correlated to the navigational screenshots. Remnant location was classified in relation to the tumor cavity: in the vertical plane as superficial, subcortical, and deep; and in the horizontal plane as anterior, medial, posterior, and lateral. Further, the relation of remnants to the retractor and to the visual axis of the surgeon, as well as the role of navigational accuracy and brain shift, were assessed. The possible reason why the remnant was not detected was then analyzed.

\section{Operative setup}

All cases have been operated at the INI Brain Suite, equipped with a high-field open-bore 1.5-T MRI scanner (MAGNETOM Espree, Siemens, Erlangen, Germany) and an integrated neuronavigation system (Vector Vision Sky 
Navigation System, BrainLAB, Heimstetten, Germany). The details regarding the setup, patient positioning, transport system, and the head fixation device, with an integrated MRI scan coil and reference markers for an automated registration procedure for navigation, have been described previously $[11,13]$. In 32 cases, functional neuronavigation was utilized: both DTI and fMRI were used in 30 cases while only DTI was considered sufficient in two cases. The first iopMR examination was performed in general anaesthesia prior to skin incision and included a standard imaging protocol for glioma surgery: T2-weighted turbo spin echo, fluid attenuated inversion recovery, and T1-weighted spin echo. Tumor remnants of grade II gliomas were evaluated on both $\mathrm{T} 1$ - and $\mathrm{T} 2$ weighted sequences. In case of a suspected remnant, its margins have been segmented and transferred into the updated navigation. These data have been superimposed in the microscope image onto the surface of the brain and/or the suspicious area was localized using the pointer system. The remnant has been removed and was sent for preliminary evaluation and for definitive histological examination. A final imaging was done for verification of the extent of definite tumor removal.

\section{Results}

In all cases, the intraoperatively acquired images were of high quality. Unexpected remnants were found in 16 patients (46\%; Table 1). The overall number of remnants was 24 because more than one site of tumor remnants was found in some patients (Table 2). In two patients, the remnants (four remnants) involved highly eloquent structures but in 14 (20 remnants) the tumor could be resected further. Complete tumor removal after additional resection could be achieved in six patients and incomplete in the remaining eight patients. Intraoperative imaging demonstrated eight remnants in the former and 16 initial remnants in the second group.

The evaluation of distribution patterns of the remnants allowed categorizing them into four groups, groups I-IV (Fig. 1):

- Group I: loss of accuracy of the navigational guidance due to brain shift

- Group II: remnant under the retractor:

(a) superficial or subcortical located remnant

(b) deep located remnant

- Group III: hidden remnant by overlapping brain

- Group IV: not obvious reason

Most frequently - in seven cases (29\%) - the remnants were not detected due to loss of accuracy of the navigational guidance as a result of brain shift (group I) -
Fig. 2. In five cases (21\%), the remnant was in the superficial or subcortical part of the resection cavity, where the retractor had been placed initially; group IIa (Fig. 3). Four patients had temporomesial grade II gliomas or tumors that extended into that region. The goal of surgery in these cases was to remove selectively the temporomesial part in order to decompress adequately the brain stem and to control the existing epilepsy. In three of them (75\% of all such tumors), the remnant was located posterolateral to the resection cavity and in one (25\%) it was superior to it (Fig. 4). These deeply located remnants were also hidden by the retractor (group $\mathrm{IIb}-16.7 \%$ of all remnants). In three cases $(13 \%)$ the small remnants were hidden by the overlapping brain (Fig. 5) - group III, while in five cases (21\%), the reason was not obvious (group IV).

Remnants of tumors that were ultimately completely removed were initially not detected due to their location under the retractor $(56 \%)$ or due to the brain shift $(22 \%$; Table 1). Remnants of incompletely resectable tumors were not detected most frequently because of loss of navigational accuracy due to brain shift $(38 \%)$.

\section{Discussion}

In the last decades, various treatment concepts of grade II gliomas have been recommended [8, 24-27, 29]. Mounting evidences demonstrate, however, that complete resection may lead to prolonged survival, delayed recurrence, and delayed malignant degeneration [2, 16, 26, 28]. The beneficial effect of radical surgery seems to be directly related to the quality of resection and the volume of residual tumor left behind [7]. In a retrospective cohort study of 170 patients with hemispheric low-grade gliomas, Mc Girth et al. [19] showed that complete resection versus subtotal resection was associated with prolonged survival. The 5-year overall survival after complete removal, neartotal removal, and subtotal removal was $95 \%, 80 \%$, and $70 \%$, respectively, and the 10-year overall survival was $76 \%, 57 \%$, and $49 \%$, respectively. After complete removal, near-total removal and subtotal removal, the median time to tumor progression was 7.0, 4.0, and 3.5 years, respectively, and the median time to malignant degeneration was 12.5 , 5.8, and 7 years, respectively. Sanai and Berger [26] reviewed all major clinical publications since 1990 on the role of extent of resection for outcome and concluded that despite persistent limitations in the quality of data, extensive surgical resection is associated with longer life expectancy for both low- and high-grade gliomas.

Moreover, some volumetric analysis suggest that the survival is related to the amount of residual tumor tissue and more aggressive resection does predict significant improvement in survival compared with a simple debulking 
Table 1 Patients with remnants: characteristics of tumor type, number and location of the remnants

\begin{tabular}{|c|c|c|c|c|c|c|c|c|}
\hline Patient & Age & Sex & Tumor type & Tumor location & $\begin{array}{l}\text { Number } \\
\text { of remnants } \\
(n=24)\end{array}$ & Type of remnant & $\begin{array}{l}\text { Radicality } \\
\text { of removal }\end{array}$ & $\begin{array}{l}\text { New neurological } \\
\text { deficit }\end{array}$ \\
\hline 1 & 38 & $\mathrm{~F}$ & Oligoastrocytoma & Frontotemporal, insular & 2 & $\begin{array}{l}\text { 1-Deep under retractor } \\
1 \text {-Hidden by overlapping brain }\end{array}$ & Incomplete & No \\
\hline 2 & 16 & $\mathrm{~F}$ & Astrocytoma & Postcentral & 1 & Loss of NN accuracy & Incomplete & No \\
\hline 3 & 40 & M & Oligoastrocytoma & Temporal, insular & 2 & Superficial under retractor & Incomplete & No \\
\hline 4 & 19 & F & Astrocytoma & Temporomesial & 1 & Deep under retractor & Complete & No \\
\hline 5 & 37 & M & Astrocytoma & Temporomesial & 2 & $\begin{array}{l}\text { 1-Deep under retractor } \\
1-\text { No obvious reason }\end{array}$ & Complete & No \\
\hline 6 & 48 & M & Astrocytoma & Temporomesial & 1 & Deep under retractor & Complete & No \\
\hline 7 & 24 & M & Astrocytoma & Frontal & 1 & Loss of NN accuracy & Incomplete & No \\
\hline 8 & 37 & M & Astrocytoma & Frontolateral & 2 & 2 -Superficial under retractor & Complete & No \\
\hline 9 & 40 & M & Oligodendroglioma & Frontoparietotem-poral, insular & 2 & $\begin{array}{l}1 \text { - Loss of NN accuracy } \\
1 \text { - No obvious reason }\end{array}$ & Incomplete & Transient hemiparesi \\
\hline 10 & 43 & M & Oligoastrocytoma & Frontal & 1 & Hidden by overlapping brain & Incomplete & No \\
\hline 11 & 41 & M & Oligodendroglioma & Frontal & 1 & Hidden by overlapping brain & Complete & No \\
\hline 12 & 23 & M & Oligodendroglioma & Temporal & 2 & Loss of NN accuracy & Complete & No \\
\hline 13 & 35 & M & Astrocytoma & Frontal & 1 & No obvious reason & Incomplete & No \\
\hline 14 & 37 & M & Oligodendroglioma & Precentral & 1 & Superficial under retractor & Incomplete & No \\
\hline 15 & 44 & M & Astrocytoma & Postcentral & 2 & 2 - Loss of NN accuracy & Incomplete & No \\
\hline 16 & 41 & $\mathrm{~F}$ & Astrocytoma & Precentral & 2 & $2-$ No obvious reason & Incomplete & Hemiparesis \\
\hline
\end{tabular}

$N N$ neuronavigation

In patients $\mathrm{N} \# 15$ and $\mathrm{N} \# 16$, the remnants involved highly eloquent structures and therefore no further resection was possible

$[15,28]$. Duffau et al. [10] also demonstrated that the death rate was significantly lower in case of subtotal and total glioma removal, in comparison with partial removal. If a residual tumor is left, it may degenerate to more malignant tumor and thus limit the survival of the patient [7].

Although many authors agree on the concept of maximal safe tumor resection, the extent of resection may be limited by the location of the glioma; it may involve or be in dangerous proximity to highly eloquent cortical and subcortical areas, to blood vessels, or to risky areas such as anterior perforated substance $[10,21]$. Thus, in our experience only $51.4 \%$ of the grade II gliomas were considered completely removable preoperatively. In such cases, however, the extent of resection may be limited by the difficulty to discern the pathological from normal brain tissue during the surgery [1]. Even an extended resection with removal of the surrounding "tumor-free" brain areas cannot guarantee that all parts of the glioma will be included because initially the tumor margins cannot be identified with certainty.

Various intraoperative imaging tools have been introduced recently that allow for more radical and safe surgeries. They provide an objective estimation of the surgical actions and thus overcome the dependence on the subjective estimation of the surgeon [7]. The ALA technique enables superior resections in malignant gliomas compared to conventional microneurosurgery [30]. In case of grade II gliomas, however, its role is rather limited.

Table. 2 Type of tumor remnants in completely and incompletely resectable tumors.

\begin{tabular}{llll}
\hline Groups & $\begin{array}{l}\text { Remnants in completely } \\
\text { removable grade II gliomas, } \% \\
(n=9)\end{array}$ & $\begin{array}{l}\text { Remnants in incompletely } \\
\text { removable grade II gliomas, \% } \\
(n=15)\end{array}$ & $\begin{array}{l}\text { Remnants in the } \\
\text { whole group, } \%, \\
(n=24)\end{array}$ \\
\hline I-Loss of accuracy of neuronavigation due to brain shift & $22.2 \%[2]$ & $33.3 \%[5]$ & $29.2 \%[7]$ \\
II-Remnant under the retractor: & $22.2 \%[2]$ & $20 \%[3]$ & $20.8 \%[5]$ \\
IIa: superficial or subcortical & $33.3 \%[3]$ & $6.7 \%[1]$ & $16.7 \%[4]$ \\
IIb: deep & $11.1 \%[1]$ & $13.3 \%[2]$ & $26.7 \%[4]$ \\
III-Hidden by overlapping brain & $11.1 \%[1]$ & $2.5 \%[3]$ \\
IV-No obvious reason & & & $20.8 \%[5]$ \\
\hline
\end{tabular}


Fig. 1 Groups of remnants location
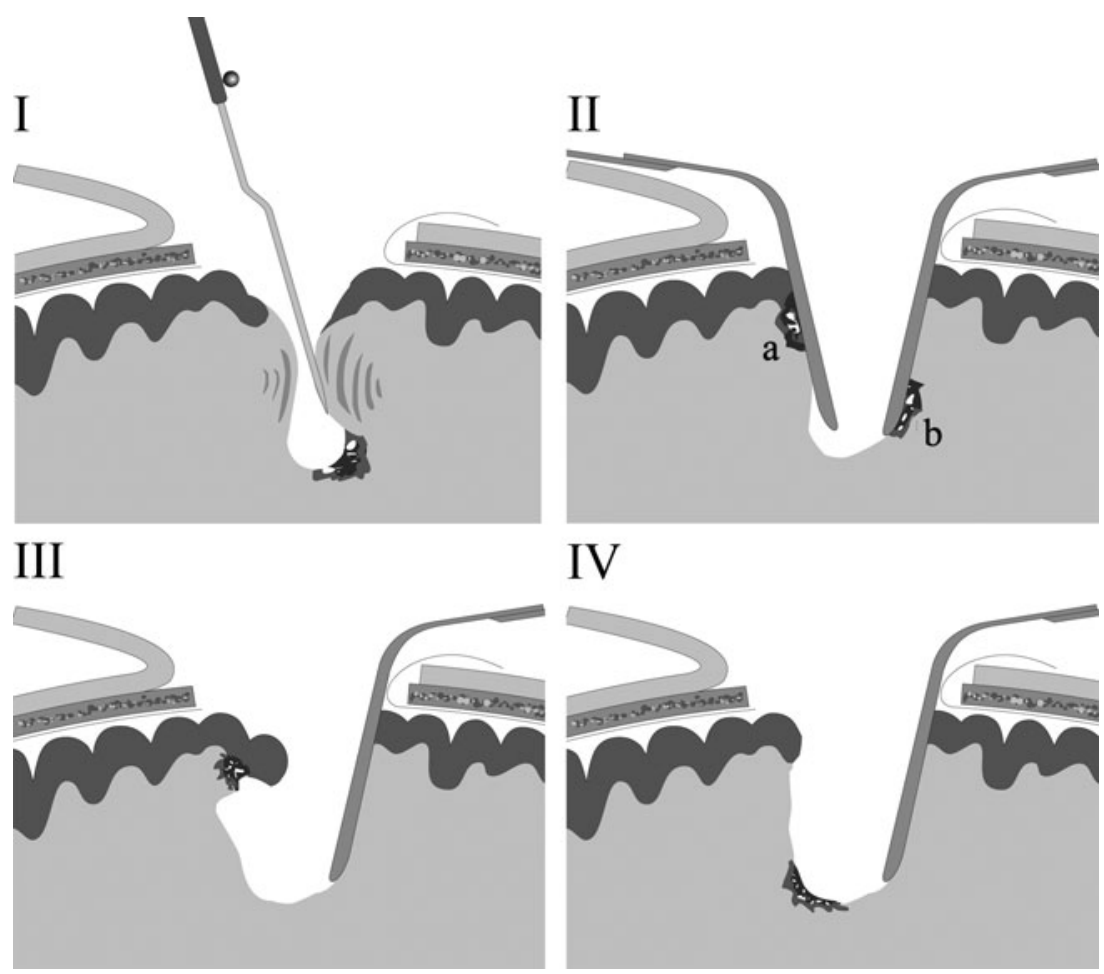

Similarly, the intraoperative CT has low soft tissue contrast and overlying artifacts if applied for grade II gliomas [32]. Neuronavigation and functional neuronavigation systems maximize the resection of all cerebral tumors but-due to the phenomenon of brain shift-become inaccurate in the course of surgery, unless an update of the preoperative data is performed [5, 23]. The ultrasound, used in a 2D mode or as $3 \mathrm{D}$ ultrasound-based neuronavigation, is a reliable tool
Fig. 2 Group I. a T1-weighted image of a relatively small frontal subcortical glioma; b microscopic view of the tumor cavity after the assumed complete tumor removal. The surgeon did not follow the tumor outlines presented by the navigation because of the expected effect of the brain shift. However, an incorrect assumption was made that the shift is identical in the superficial and deeper parts of the brain. A remnant $(\mathrm{R})$ was identified deep and posterior to the resection cavity (c); d complete tumor removal. A small blood clot is seen in the resection cavity. The comparison with the preoperative images showed that the hyperintense areas around the resection cavity are grey matter
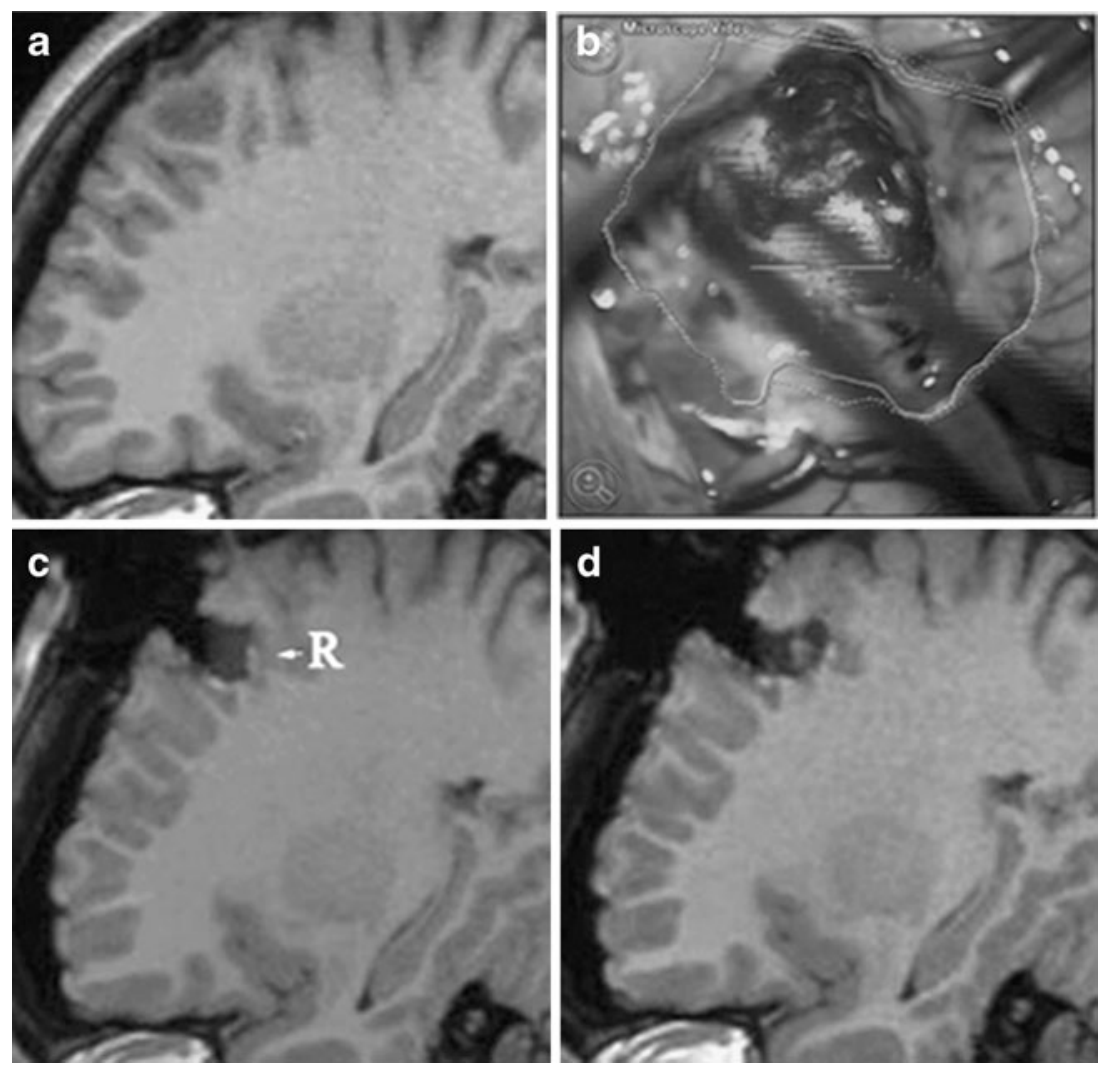
Fig. 3 Group II a: the patient is positioned with the head straight on the operating table. a preoperative view of the tumor; the remnant is located posterior to the resection cavity, where the brain retractor was placed initially (b); c intraoperative view, presenting the location of the remnant ( $R$ remnant, $F$ falx, $a$ anterior, $p$ posterior); $\mathbf{d}$ final MRI proving the complete tumor removal
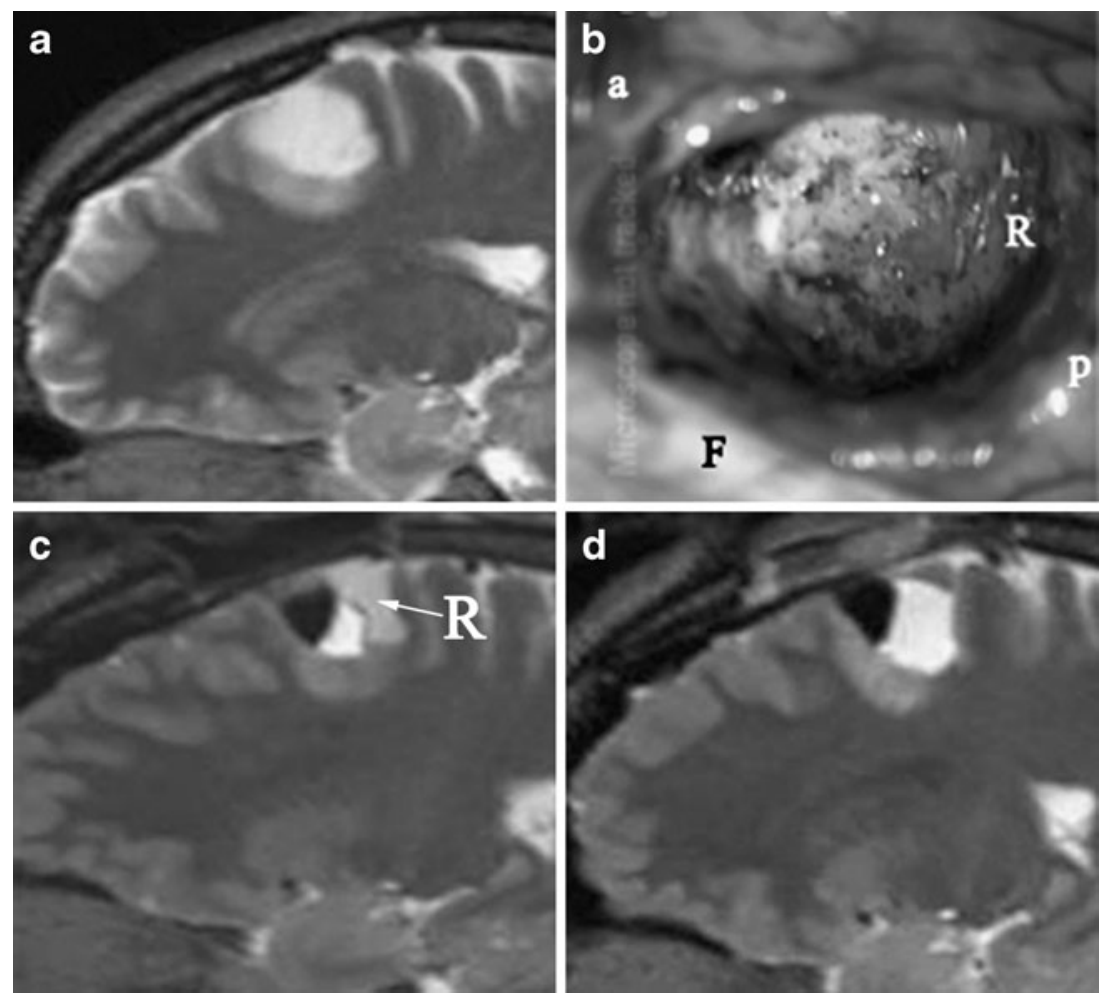

even in grade II gliomas and provides almost instant realtime feedback information [33,34]. Further, it is easier to handle, less time consuming, and less costly than the iopMRI. Its accuracy is highest in case of more confined deeply located remnants but in case of more superficially located remnants its role is more limited [13].

The high-field iopMRI is the current golden standard in intraoperative imaging $[6,11,14,20]$. It offers the greatest range in cranial imaging with a quality that parallels those of the diagnostic images. The iopMRI allows for detection of even small tumor remnants and for compensation of the brain shift by providing updates of the preoperative anatomical and functional neuronavigational data $[6,11$, $12,18,22]$. Thus, more complete tumor removal can be achieved. In a recent series of 49 patients with gliomas, the iopMR imaging reveled residual tumor in $80 \%$. In 19 patients, further resection could be performed and gross total removal was achieved in $35 \%$. Of these patients in whom resection was complete, $41 \%$ underwent further resection after iopMR imaging [20]. Furthermore, a significant reduction in residual tumor volume was demonstrated in those cases that could not be removed completely: the residual tumor volume decreased from $26.5 \%$ to $11.6 \%$ in patients with intended partial
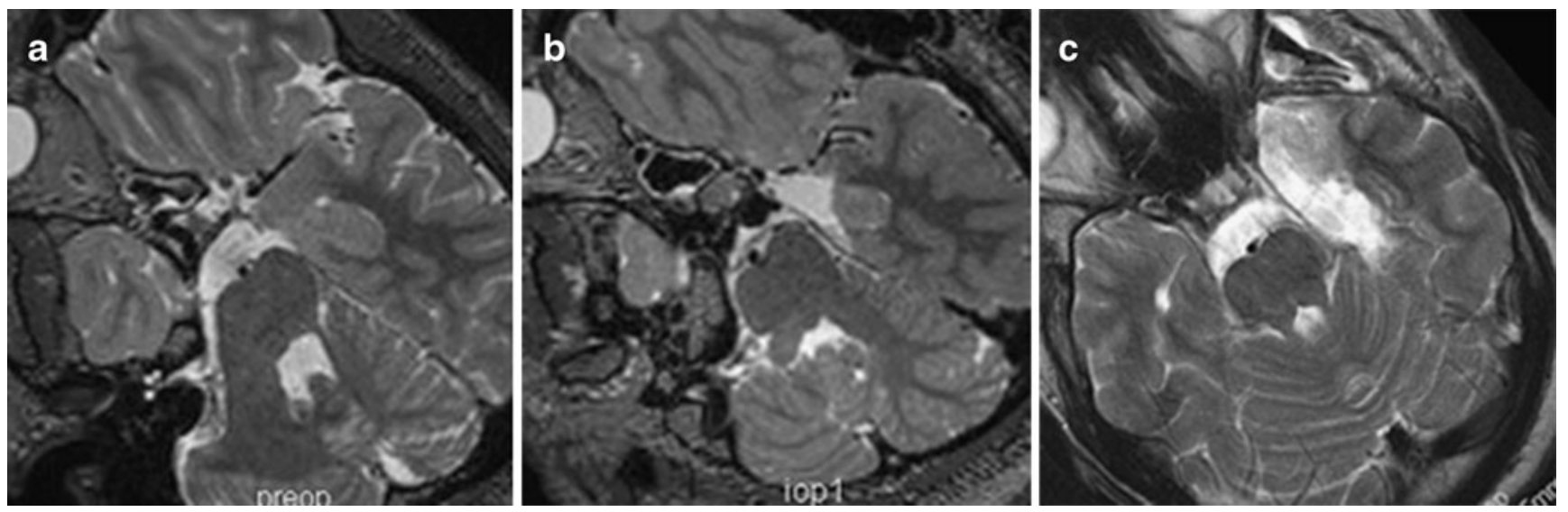

Fig. 4 In temporomesial tumors, the remnant is frequently found posterolaterally to the resection cavity (Group IIb). a Preoperative view of the tumor; b view after the assumed complete removal. The remnant (R) is well visualized; c follow-up MRI 6 months after the complete tumor removal. (Figures $\mathbf{a}$ and $\mathbf{b}$ are intraoperative images and the head tilt is different from that on the follow up image c) 
Fig. 5 Group III: this small remnant, which is outlined on the figures, has not been seen during the surgery due to poor visualization of this portion of the resection cavity - it has been partially hidden by the overlapping gyrus. Fig. 4a: neuronavigation screenshot ( $a$ anterior, $b$ posterior); Fig. 4b: intraoperative microscopic view with superimposed outlines of the residual tumor
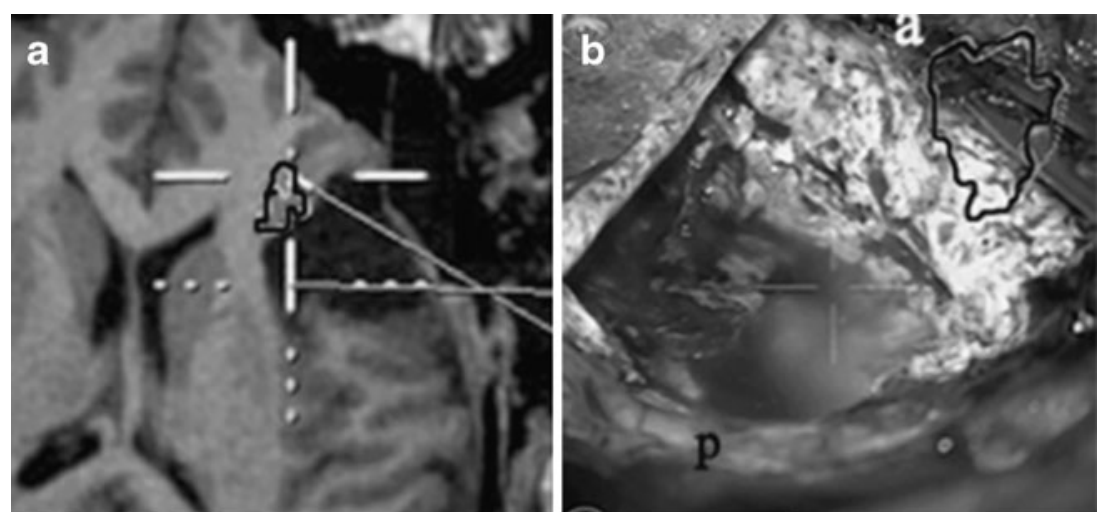

resection. The iopMRI may increase the survival; Claus et al. [9] evaluated 156 patients with grade II gliomas who underwent surgery with iopMRI guidance. They showed that the patients who underwent partial resection were at 1.4 times the risk of recurrence and 4.9 times the risk of death as patients who underwent total resection. The widespread utilization iopMRI, however, is limited by its very high cost, more complex logistical setup, and disturbance of the operative workflow.

Systematic evaluation of tumor remnants may give clues how to achieve more radical surgeries. Manndonet et al. [17] described the zones in which tumor residual is most frequently found. Although the study was based on MR imaging performed 3 months after surgery, the slow progress of grade II gliomas justified this approach. The authors found that remnants were most frequently close to eloquent cortical or subcortical areas or involved risky areas, such as anterior perforated substance and posterior corpus callosum, e.g., those remnants could be predicted due to their anatomical location. Talos et al. [31] examined the variables for incomplete tumor resection in a series of 101 patients with grade II gliomas. Their analysis showed that certain preoperatively available factors correlated with the outcome: tumor volume, tumor type, tumor margin on T2-weighted MRI, and involvement of eloquent areas.

In our study, however, we focused on those intraoperative factors that influence the radicality of surgery but cannot be predicted. We found unexpected remnants in $46 \%$ of the patients and demonstrated that their spatial distribution follows some patterns. These patterns are not related to the tumor type or size but rather on its $3 \mathrm{D}$ location and its relation to surrounding brain structures. The avoidance of neurological deficits has been always of paramount importance and-expectedly-the remnants expected by the surgeon were most frequently left due to their proximity to highly eloquent brain areas: motor cortex, speech areas, or major fiber tracts. The neuronavigational guidance becomes inaccurate during tumor removal and the surgeon tends to leave a sufficient safety margin to the essential structures.
During surgery, varying amount of brain shift develops resulting from brain deformation caused by patient positioning or use of brain retractors, loss of cerebrospinal fluid, and tumor debulking. These changes could be predicted at least to some extent and the surgeon can modify his technique accordingly. The expected effect of the brain shift, however, was found to be often overestimated. In our study, we found that inaccuracy of navigation due to brain shift was the most frequent reason for leaving remnants after assumed by the surgeon complete removal - in 36.8\% of all cases. The plasticity of the brain is heterogeneous and the brain shift is more pronounced in the superficial parts of the resection cavity - it collapses more than the deeper part (Fig. 1). Unless an intraoperative update is performed, the amount and direction of the shift cannot be predicted, and such remnants (group I of our classification) cannot be removed.

\section{Lessons learned}

In $21 \%$ of our cases, the remnant was found in the posterior part of the resection cavity, towards the surgeon, where the retractor had been placed initially. Even though the retractor was later shifted and the area beneath it is inspected, the observed changes were assumed falsely to be retractor related. In 13\%, a small residual was not identified because it was hidden by the overlapping brain. The use of a retractor should be, therefore, avoided whenever possible. In case of deeply located tumors, which cannot be accessed without its support, a focused attention to the areas of retraction, as well as the systematic inspection of all parts of the resection cavity for remaining tumor tissue, may increase the radicality of surgery. Surgical resection of temporomesial tumors or of the temporomesial part of more extensive gliomas in order to control epilepsy or decompress the cerebral peduncle is relatively common procedure [4]. Their anterior and posteromedial parts can be removed completely and the extent of resection could be reliably guided by the existing anatomical landmarks. In this study, we found that remnants of such tumors were located 
typically posterolateral to the resection cavity-in $75 \%$ of these cases. Knowledge of these three groups and a targeted attention to the corresponding brain areas, may lead to more radical removal even without intraoperative imaging control. In case of superficial tumors, the use of retractor should be avoided; however, in deep-seated tumors this is essential. In cases retractor has to be used, the areas bellow it should be inspected thoroughly. Ideally, it should be always placed at the resection border between the tumor and the normal brain, if it can be determined at all.

\section{Conclusion}

Distribution of tumor remnants of grade II gliomas tends to follow some patterns. The remnants were not detected due to loss of accuracy of the navigational guidance or were hidden by the retractor or overlying cortex. Targeted attention to these areas could increase the radicality of surgery even if intraoperative imaging is not performed.

\section{Acknowledgment}

Disclosure Parts of this study have been presented at the DGNC meeting in Münster, May 25-27, 2009.

\section{Conflicts of interest None.}

\section{References}

1. (1998) Practice parameters in adults with suspected or known supratentorial nonoptic pathway low-grade glioma. Neurosurg Focus 4:e10

2. Bauman G, Fisher B, Watling C, Cairncross JG, Macdonald D (2009) Adult supratentorial low-grade glioma: long-term experience at a single institution. Int J Radiat Oncol Biol Phys 75:1401-1407

3. Berger MS, Deliganis AV, Dobbins J, Keles GE (1994) The effect of extent of resection on recurrence in patients with low grade cerebral hemisphere gliomas. Cancer 74:1784-1791

4. Berger MS, Hadjipanayis CG (2007) Surgery of intrinsic cerebral tumors. Neurosurgery 61:279-304, discussion 304-275

5. Berger MS, Rostomily RC (1997) Low grade gliomas: functional mapping resection strategies, extent of resection, and outcome. J Neurooncol 34:85-101

6. Black PM, Alexander E 3rd, Martin C, Moriarty T, Nabavi A, Wong TZ, Schwartz RB, Jolesz F (1999) Craniotomy for tumor treatment in an intraoperative magnetic resonance imaging unit. Neurosurgery 45:423-431, discussion 431-423

7. Bradley WG (2002) Achieving gross total resection of brain tumors: intraoperative MR imaging can make a big difference. AJNR Am J Neuroradiol 23:348-349

8. Cavaliere R, Lopes MB, Schiff D (2005) Low-grade gliomas: an update on pathology and therapy. Lancet Neurol 4:760-770

9. Claus EB, Horlacher A, Hsu L, Schwartz RB, Dello-Iacono D, Talos F, Jolesz FA, Black PM (2005) Survival rates in patients with low-grade glioma after intraoperative magnetic resonance image guidance. Cancer 103:1227-1233
10. Duffau H, Lopes M, Arthuis F, Bitar A, Sichez JP, Van Effenterre R, Capelle L (2005) Contribution of intraoperative electrical stimulations in surgery of low grade gliomas: a comparative study between two series without (1985-96) and with (1996-2003) functional mapping in the same institution. J Neurol Neurosurg Psychiatry 76:845-851

11. Fahlbusch R, Samii A (2007) A review of cranial imaging techniques: potential and limitations. Clin Neurosurg 54:100-104

12. Ganser KA, Dickhaus H, Staubert A, Bonsanto MM, Wirtz CR, Tronnier VM, Kunze S (1997) Quantification of brain shift effects in MRI images. Biomed Tech 42:247-248

13. Gerganov VM, Samii A, Akbarian A, Stieglitz L, Samii M, Fahlbusch R (2009) Reliability of intraoperative high-resolution $2 \mathrm{D}$ ultrasound as an alternative to high-field strength MR imaging for tumor resection control: a prospective comparative study. J Neurosurg 111:512-519

14. Hatiboglu MA, Weinberg JS, Suki D, Rao G, Prabhu SS, Shah K, Jackson E, Sawaya R (2009) Impact of intraoperative high-field magnetic resonance imaging guidance on glioma surgery: a prospective volumetric analysis. Neurosurgery 64:1073-1081, discussion 1081

15. Karim AB, Maat B, Hatlevoll R, Menten J, Rutten EH, Thomas DG, Mascarenhas F, Horiot JC, Parvinen LM, van Reijn M, Jager JJ, Fabrini MG, van Alphen AM, Hamers HP, Gaspar L, Noordman E, Pierart M, van Glabbeke M (1996) A randomized trial on dose-response in radiation therapy of low-grade cerebral glioma: European organization for research and treatment of cancer (EORTC) study 22844. Int J Radiat Oncol Biol Phys 36:549-556

16. Keles GE, Lamborn KR, Berger MS (2001) Low-grade hemispheric gliomas in adults: a critical review of extent of resection as a factor influencing outcome. J Neurosurg 95:735-745

17. Mandonnet E, Jbabdi S, Taillandier L, Galanaud D, Benali H, Capelle L, Duffau H (2007) Preoperative estimation of residual volume for WHO grade II glioma resected with intraoperative functional mapping. Neuro Oncology 9:63-69

18. Maurer M, Becker G, Wagner R, Woydt M, Hofmann E, Puls I, Lindner A, Krone A (2000) Early postoperative transcranial sonography (TCS), CT, and MRI after resection of high grade glioma: evaluation of residual tumour and its influence on prognosis. Acta Neurochir (Wien) 142:1089-1097

19. McGirt MJ, Chaichana KL, Attenello FJ, Weingart JD, Than K, Burger PC, Olivi A, Brem H, Quinones-Hinojosa A (2008) Extent of surgical resection is independently associated with survival in patients with hemispheric infiltrating low-grade gliomas. Neurosurgery 63:700-707, author reply 707-708

20. Nimsky C, Fujita A, Ganslandt O, Von Keller B, Fahlbusch R (2004) Volumetric assessment of glioma removal by intraoperative high-field magnetic resonance imaging. Neurosurgery 55:discussion 370-351-370

21. Nimsky C, Ganslandt O, Buchfelder M, Fahlbusch R (2003) Glioma surgery evaluated by intraoperative low-field magnetic resonance imaging. Acta Neurochir Suppl 85:55-63

22. Nimsky C, Ganslandt O, Buchfelder M, Fahlbusch R (2006) Intraoperative visualization for resection of gliomas: the role of functional neuronavigation and intraoperative 1.5 T MRI. Neurol Res 28:482-487

23. Nimsky C, Ganslandt O, Cerny S, Hastreiter P, Greiner G, Fahlbusch R (2000) Quantification of, visualization of, and compensation for brain shift using intraoperative magnetic resonance imaging. Neurosurgery 47:1070-1079, discussion 1079-1080

24. Philippon JH, Clemenceau SH, Fauchon FH, Foncin JF (1993) Supratentorial low-grade astrocytomas in adults. Neurosurgery 32:554-559

25. Pouratian N, Asthagiri A, Jagannathan J, Shaffrey ME, Schiff D (2007) Surgery Insight: the role of surgery in the management of low-grade gliomas. Nat Clin Pract Neurol 3:628-639 
26. Sanai N, Berger MS (2008) Glioma extent of resection and its impact on patient outcome. Neurosurgery 62:753-764, discussion 264-756

27. Shaw EG, Berkey B, Coons SW, Bullard D, Brachman D, Buckner JC, Stelzer KJ, Barger GR, Brown PD, Gilbert MR, Mehta M (2008) Recurrence following neurosurgeon-determined gross-total resection of adult supratentorial low-grade glioma: results of a prospective clinical trial. J Neurosurg 109:835-841

28. Smith JS, Chang EF, Lamborn KR, Chang SM, Prados MD, Cha S, Tihan T, Vandenberg S, McDermott MW, Berger MS (2008) Role of extent of resection in the long-term outcome of low-grade hemispheric gliomas. J Clin Oncol 26:1338-1345

29. Soffietti R, Chio A, Giordana MT, Vasario E, Schiffer D (1989) Prognostic factors in well-differentiated cerebral astrocytomas in the adult. Neurosurgery 24:686-692

30. Stummer W, Pichlmeier U, Meinel T, Wiestler OD, Zanella F, Reulen HJ (2006) Fluorescence-guided surgery with 5 -aminolevulinic acid for resection of malignant glioma: a randomised controlled multicentre phase III trial. Lancet Oncol 7:392-401
31. Talos IF, Zou KH, Ohno-Machado L, Bhagwat JG, Kikinis R, Black PM, Jolesz FA (2006) Supratentorial low-grade glioma resectability: statistical predictive analysis based on anatomic MR features and tumor characteristics. Radiology 239:506513

32. Uhl E, Zausinger S, Morhard D, Heigl T, Scheder B, Rachinger W, Schichor C, Tonn JC (2009) Intraoperative computed tomography with integrated navigation system in a multidisciplinary operating suite. Neurosurgery 64:231-239, discussion 239-240

33. Unsgaard G, Rygh OM, Selbekk T, Muller TB, Kolstad F, Lindseth F, Hernes TA (2006) Intra-operative 3D ultrasound in neurosurgery. Acta Neurochir 148:235-253, discussion 253

34. Unsgaard G, Selbekk T, Brostrup Muller T, Ommedal S, Torp SH, Myhr G, Bang J, Nagelhus Hernes TA (2005) Ability of navigated 3D ultrasound to delineate gliomas and metastases-comparison of image interpretations with histopathology. Acta Neurochir (Wien) 147:discussion 1269-1269 